\title{
Das Junge Forum der DGOU - Über die Wünsche und Vorschläge des Nachwuchses in Unfallchirurgie und Orthopädie
}

The Young Forum of the DGOU - On the Wishes and Suggestions of the Next Generation in Trauma Surgery and Orthopaedics

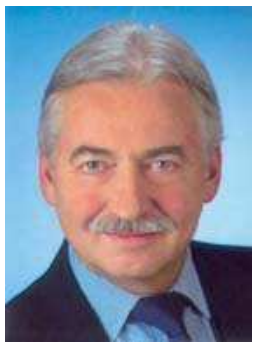

K. Weise

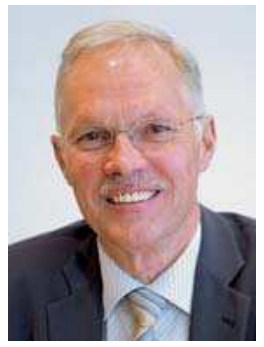

F. U. Niethard
„Wer operiert einmal meinen Schenkelhalsbruch und wer versorgt mich mit einer Endoprothese, wenn ich im Alter das eine oder das andere benötigen sollte."

Solche oder so ähnliche, meist in scherzhaftem Unterton geäußerte Verlautbarungen sind in der Generation von gestandenen, der beruflichen Altersgrenze entgegengehenden Kollegen unserer Fachgebiete immer häufiger zu vernehmen, basierend auf der Befürchtung, die heutigen Rahmenbedingungen im Gesundheitswesen allgemein und die speziellen Belastungen bei der Ausübung chirurgischer Tätigkeit könnten einen anhaltenden Nachwuchsmangel begründen. Die erfreuliche Statistik aktueller Neuaufnahmen von Mitgliedern in DGU und DGOOC aus dem Kreis nachrückender Orthopäden und Unfallchirurgen scheint dieser Befürchtung zu widersprechen, andererseits existieren valide Zahlen, die eine ganz andere Sprache sprechen. In einer Publikation von M. Krüger aus Magdeburg, veröffentlicht in den Mitteilungen des BDC 9/2008 über die chirurgische Weiterbildung aus Assistentensicht ist von einem seit 1991 um 40\% gesunkenen Anteil jüngerer Ärzte die Rede. Nur noch 5\% der Studierenden können sich eine Weiterbildung in einem chirurgischen Fach vorstellen, was womöglich auch mit dem bis an die $70 \%$ reichenden Anteil weiblicher Studierender pro Semester und der Tatsache zu tun hat, dass der Anteil von Ärztinnen in der Chirurgie zwar allmählich ansteigt, jedoch traditionell niedriger ist als in medizinischen Fächern, die als frauen- und familienfreundlicher gelten. Der Einstieg in eine der chirurgischen Disziplinen kommt einer weiteren Erhebung zufolge nur noch für 5\% der Studierenden überhaupt infrage.

Was also ist zu tun, will man die Attraktivität der Chirurgie im Allgemeinen und diejenige von Unfallchirurgie und Orthopädie im Besonderen in dem Maße erhöhen, dass Schlagzeilen wie „Von der Ärzteschwemme zum Ärztemangel“, „Ärztemangel trotz Zuwachsraten“, „Die Migration von Ärzten“ und „Nachwuchssorgen in der Chirurgie“ der Vergangenheit angehören? Die dafür geeigneten Instrumente sind tatsächlich schon seit Längerem bekannt, werden aber augenscheinlich noch nicht in ausreichender Konsequenz und flächendeckend zur Anwendung gebracht. Bereits im Jahre 2002 wurden im „Deutschen Ärzteblatt“ unter dem Titel „Nachwuchssorgen - Chirurgie“ diejenigen Problembereiche benannt, in welchen durch Anpassung und Optimierung der bestehenden Verhältnisse an die Wünsche und Vorstellungen des chirurgischen Nachwuchses gearbeitet werden müsste, um das erwartete Defizit an chirurgisch tätigen Ärzten in den kommenden Jahren zu vermeiden. Die dazugehörigen Themen umfassten die Verbesserung der Zukunftsperspektiven, das Schaffen akzeptabler Arbeitsbedingungen in den Krankenhäusern und eine planbare Weiterbildung.

Für die Fachgesellschaften DGU und DGOOC ist das Erfordernis, die nachrückende Generation an Unfallchirurgen und Orthopäden, nicht zuletzt vor dem Hintergrund des gemeinsamen Facharztes und der zu dessen Erlangung etablierten Weiterbildung, nachhaltig in die berufs- und gesundheitspolitischen Aktivitäten einzubinden permanente Verpflichtung, um zusammen mit dem Nachwuchs günstige Perspektiven für die Zukunft zu eröffnen sowie die dafür erforderlichen Rahmenbedingungen zu schaffen. Das aus den Reihen jüngerer Mitglieder der DGU im Jahr 2004 hervorgegangene Junge Forum hat sich auf die Fahnen geschrieben, die Aktivitäten auf die formulierten Arbeitsschwerpunkte zu konzentrieren und auf eine strukturierte Umsetzung der Weiterbildung zum gemeinsamen Facharzt hinzuarbeiten, um noch bestehende Unsicherheiten zu eliminieren und damit die Attraktivität unseres Berufsbilds zu erhöhen [1]. Eine seit 2005 eingerichtete Stellenbörse arbeitet erfolgreich an der Vermittlung von Beschäftigungsverhältnissen über das Internet, der vom Jungen Forum entworfene Flyer dient der Mitgliederwerbung und möchte den medizinischen Nachwuchs für die Unfallchirurgie begeistern. Ein weiteres Ziel in den Bestrebungen des Jungen Forums ist es, die Bedeutung wissenschaftlichen Engagements in der Unfallchirurgie schon während der studentischen Ausbildung, ganz besonders aber im Rahmen der fachärztlichen Weiterbildung zu verdeutlichen [2].

Seit 2007 existiert das gemeinsame Junge Forum der Orthopäden und Unfallchirurgen als Nachwuchsorganisation der DGOU, um die gemeinsamen Interessen der nachrückenden Generation in Weiterbildung befindlicher Ärzte zu bündeln. Die Zeitschrift für Orthopädie und Unfallchirurgie bietet dem Jungen Forum eine Plattform, um Informationen weiterzugeben und auf interessante Projekte hinzuweisen [3]. Zu diesen zählt beispielsweise die „Summerschool Orthopädie und 
Unfallchirurgie“ ebenso wie die Ausschreibung eines „Förderpreises des Jungen Forums der DGOU“ und nicht zuletzt die Mitwirkung an der Programmgestaltung des DKOU 2008 [4,5]. Zudem sind Frau Dr. Witte (DGOOC) und PD Dr. Matthes (DGU) Mitglieder im Nichtständigen Beirat der DGOU, um dort die abgestimmten Vorstellungen und Wünsche des unfallchirurgisch-orthopädischen Nachwuchses artikulieren zu können.

Erste wichtige Schritte in die richtige Richtung sind bereits getan, den Nachwuchs in Orthopädie und Unfallchirurgie sichern zu helfen, indem die Rahmenbedingungen für eine strukturierte Weiterbildung und die Situation am Arbeitsplatz Krankenhaus kontinuierlich verbessert, mithin günstigere Zukunftsperspektiven eröffnet und damit die Attraktivität unserer Fachgebiete veranschaulicht werden. Dies könnte und sollte nicht zuletzt auch Auswirkungen auf eine stetige Erhöhung der Zahl an Ärztinnen in Weiterbildung zur Facharztkompetenz „Orthopädie und Unfallchirurgie“ haben. Die Herausgeber der Zeitschrift für Orthopädie und Unfallchirurgie schätzen die Zusammenarbeit mit dem
Jungen Forum der DGOU im Bestreben der Förderung des Nachwuchses als ausgesprochen wertvoll ein und werden von ihrer Seite keine Mühen scheuen, diese Aktivitäten auch weiterhin nachhaltig zu unterstützen.

\section{K. Weise}

F. U. Niethard

\section{Literatur}

1 Witte $D$, Wölfl C. Ein Netzwerk für den orthopädisch-unfallchirurgischen Nachwuchs in Deutschland. Z Orthop Unfall 2008; 146: 298-299

2 Davids D, Reumann M, Reumann M. „Medics meets Engineers“. Z Orthop Unfall 2008; 146: 698-699

3 Matthes $G$, Witte $D$. „Neues vom Jungen Forum der Orthopäden und Unfallchirurgen". Z Orthop Unfall 2008; 146: 425

4 Matthes G et al. Sitzung des Jungen Forums. Z Orthop Unfall 2008; 146 : 699

5 Wölfl C, Witte D. Nachwuchstag des Jungen Forum in Berlin 2008. Z Orthop Unfall 2008; 146: 563 